\title{
La Administración Pública y sus clientes: ¿Moda organizativa u opción ideológica?
}

Q. Brugué, M. Amorós y R. Gomà *

"La necesidad de mejorar estas relaciones [entre los ciudadanos y la Administración] se considera hoy en todos los paises como una obligación inexcusable. Por esta causa, los gobiernos vienen acometiendo desde hace varios años actuaciones de reforma de sus Administraciones públicas, presididas por la idea de que la creencia clásica en unas relaciones descompensadas entre Administración y administrados debe dejar paso a una nueva concepción de carácter igualitario en la que la Administración no es sino un servicio y el público, su clientela." (OCDE, 1991:9)
On estas palabras se expone una de las ideas que más ha influido en la reestructuración de la Administración Pública moderna: la clientelización de las relaciones entre la Administración y sus administrados. A través de experimentos organizativos dispares y bajo la tutela intelectual de una abundante literatura, se ha impulsado y asumido la bondad del proceso. Este amplio acuerdo, sin embargo, no ha llegado acompañado de argumentos coincidentes. Desde espacios ideológicos distintos se aplaude a aquellas organizaciones que se han mostrado más cercanas a sus consumidores, que satisfacen mejor las demandas de sus clientes, o que son capaces de identificar con más precisión las necesidades de su público. Pero en este aplauso generalizado cada observador destaca matices diferentes. Existe unanimidad en relación a la idea marco, pero su articulación específica es objeto de un debate que suele sorprender, precisamente, a los que la defienden con más entusiasmo. Y suele sorprenderles porque la definición más rudimentaria de este enfoque destaca la objetividad de sus planteamientos: una vez razonada la idoneidad de las propuestas, cualquier discusión está de más.

Esta aureola de neutralidad y de inevitabilidad ha provocado, como mínimo, dos dificultades. En primer lugar, se ha trasladado a un segundo plano la tarea de conceptualizar teóricamente el propio enfoque, dejando sus términos poco definidos y sus argumentos sumidos en cierta ambigüedad. En segundo lugar, se trata de un debate que ha nacido en el seno de las organizaciones y que se ha vanagloriado en todo momento de su apoliticidad. La Administración Pública, sin embargo, no sólo produce determinados bienes y servicios, sino que lo hace en defensa de unos valores colectivos y de unos planteamientos político-ideológicos específicos. Marginar estos elementos puede generar importantes lagunas, que sólo recientemente están empezando a ser abordadas.

El objetivo de este artículo es precisamente dar respuesta a las dos dificultades a las que acabamos de referirnos. Por un lado, pretendemos resumir y ordenar las principales aportaciones al debate. Por otro lado, queremos hacerlo de forma crítica, es decir, señalando sus límites e introduciendo aquellos elementos valorativos 


\section{Cuadro 1 Cambio de modelo: variables explicativas e impactos}

Cambios en el entorno
Crisis del modelo keynesiano y burocrático
Variables económico-sociales
Impacto sobre la dimensión sustantiva

Erosión capacidad reguladora del keynesianismo.
1. Caída tasas de crecimiento. Crisis fiscal del Estado. Crisis fiscal del Estado.

2. Transformación modelo productivo.

3. Fragmentación estructura social.
Nuevos actores y nuevas demandas colectivas.
Colapso de las rigideces burocráticas. Colapso del monopolio burocrático.

Fitente Elaboracion propin.

que, en nuestra opinión, son necesarios para entender el potencial y los peligros de los enfoques que analizamos.

Con este fin, hemos estructurado el trabajo en cuatro apartados. En primer lugar, repasamos las recientes transformaciones que han experimentado las Administraciones Públicas occidentales, dedicando una especial atención a sus relaciones con las personas. En segundo lugar, agrupamos y analizando los elementos teóricos de la llamada corriente consumerista. En tercer lugar, destacamos los limites y las alternativas de este enfoque. Finalmente, tras introducir la variable político-ideológica, vinculamos el debate sobre el rol de los individuos con una discusión sobre las diferentes formas de entender la organización y la naturaleza del Estado. Recogemos algunas de las propuestas teóricas elaboradas y exponemos nuestro propio punto de vista.

\section{Políticas públicas y Administración: alcance y orientación de las transformaciones recientes}

1. Cambio de Modelo y Giro Ideológico: de la Redistribución Burocrática al Neoliberalismo Excelente

A lo largo de las dos últimas décadas, una de las ideas más ampliamente aceptadas en el campo de las ciencias sociales ha sido la crisis estructural del Modelo Clásico de Bienestar, construido en las democracias capitalistas avanzadas después de la segunda guerra mundial. Muy en síntesis, el Estado de Bienestar Keynesiano (EBK) supuso una rearticulación de las relaciones Estado-mercado, sobre la base de un amplio consenso socio-político, expresado en tres direcciones: el compromiso público con el pleno empleo estable, por medio de políticas económicas expansivas; la reproducción masiva de la población ocupada, a través de sistemas públicos de sanidad, enseñanza, transporte y vivienda; y el mantenimiento de los segmentos excluidos del mercado de trabajo, por medio de mecanismos públicos de seguridad social y garantía de rentas ${ }^{1}$.

El colapso del EBK se explica a partir de un conjunto de factores económico-sociales que presionan sobre la esfera pública (Cuadro 1). En concreto: la caída de las tasas sostenidas de crecimiento, la quiebra del modelo de producción fordista y la fragmentación de la estructura social. Dichos procesos se trasladan al sistema político en forma de tensiones fiscales, erosión de la capacidad reguladora del keynesianismo y eclosión de nuevos actores y demandas colectivas al margen de los partidos tradicionales.

Así pues, las variables económico-sociales explican la crisis del modelo y generan las presiones iniciales de cambio. Sin embargo, son los componentes político-ideológicos los que van a determinar el alcance, la naturaleza y la orientación de fondo de las transformaciones posteriores. En concreto, el consenso social-reformista es desplazado, de forma progresiva, por la hegemonía ideológica neoliberal, la cual cristaliza en dos grandes campos de la praxis política sustantiva. En el económico, por medio 
de una combinación de monetarismo, políticas de oferta y desregulaciones laborales, orientadas a la recuperación de las tasas de beneficio como motor de la reactivación global'? En el social, a través de la remercantilización de bienes y servicios públicos y la correlativa residualización de los roles redistributivos del Estado (KinG, 1987; MuÑoz DE BUSTLLO, 1989).

Todo lo anterior tiene lugar en la esfera sustantiva de la política, es decir, en las estrategias de regulación del conflicto social. No puede obviarse, sin embargo, la estrecha conexión entre dicho terreno y los modelos de Administración Pública que se configuran. En définitiva, los sistemas de gestión y organización pública reciben fuertes presiones externas y tienden a adaptarse -de forma lenta y contradictoria- a las funciones asumidas por el Estado y a las bases de legitimación político-cultural que lo sostienen.

Así pues, la crisis del EBK comporta, en la esfera operativa, el colapso del modelo socioburocrático, expresado en tres dimensiones fundamentales: la necesaria superación de sus rigideces organizativas, de su carácter monopolista y de sus relaciones de prestación, basadas en una concepción pasiva y dependiente de los usuarios de servicios. Sobre estas dimensiones se dibujan los grandes ejes del paradigma posburocrático emergente (ELCOCK, 1991; BaRZELAY, 1992). Estos, en la práctica, tienden a concretarse en: a) una organización flexible, capaz de apoyar nuevos principios de gestión pública de raíz empresarial, enmarcados en el concepto de excelencia; b) un sistema de prestación pluralista, basado en relaciones competitivas de carácter mercantil; y c) una tendencia hacia la clientelización de las relaciones de la Administración con sus públicos ${ }^{3}$.

\section{Hacia una Reinterpretación Politológica Crítica}

Lo desarrollado en el punto anterior, intenta reflejar de forma analítica las tendencias predominantes en los terrenos de la regulación y la gestión pública a lo largo de la década de los ochenta. En ese contexto, cabe señalar el hecho de que gran parte de los análisis politológicos sobre dichas cuestiones ha adoptado planteamientos acríticos. Ello ha conducido a una doble concepción, más o menos explícita, según la cual la praxis neoliberal prefigura la única alternativa de reestructuración sustantiva del EBK, y los elementos de administración posburocrática predominantes revisten un carácter éticamente neutro, ligado de forma exclusiva a racionalidades funcionales de tipo eficientista.

En los noventa, esta doble concepción ha empezado a ser revisada. En primer lugar, se pone de manifiesto el carácter no necesariamente unidireccional del proceso de redefinición sustantiva del Estado de Bienestar. Ello permite la introducción de una variable de tipo político-ideológico que dicotomiza la esfera sustantiva: frente a las opciones mercantilizadoras, se sitúa, como estrategia alternativa, la extensión y profundización de la regulación pública ${ }^{4}$. El cuadro 2 lo expresa gráficamente. El desplazamiento en el eje horizontal hacia el pluralismo posburocrático, permite, de forma simultánea, diferentes ubicaciones en el eje vertical (Brugué, 1993; Gomà, 1992).

En segundo lugar, se pone de manifiesto la conexión normativa entre el enfoque neoliberal y la posburocracia basada en la excelencia, el pluralismo competitivo y la clientelización. Se destruye así la supuesta neutralidad valorativa del paradigma posburocrático y se abre un

\begin{tabular}{|c|c|}
\hline \multicolumn{2}{|r|}{$\begin{array}{l}\text { as y operativas. } \\
\text { atico }\end{array}$} \\
\hline \multicolumn{2}{|c|}{\begin{tabular}{l|l}
\multicolumn{2}{c}{ Estado } \\
Testrategia de mantenimiento/extensión) \\
Tipos integral \\
M corporativo del EBK
\end{tabular} \mid $\begin{array}{l}\text { Diferentes tipos } \\
\text { de Estado de Bienestar } \\
\text { pluralista }\end{array}$} \\
\hline $\begin{array}{r}\text { Tipo residual } \\
\text { del EBK }\end{array}$ & $\begin{array}{l}\text { Estado Neoliberal } \\
\text { pluralista } \\
\text { ción) }\end{array}$ \\
\hline
\end{tabular}




\begin{tabular}{|c|c|c|}
\hline \\
\hline \multicolumn{3}{|c|}{$\begin{array}{c}\text { Cucudro } 3 \\
\text { Escenarios alternativos en el marco del muevo modelo } \\
\text { de Administración Pública } \\
\text { Modelo mercantil }\end{array}$} \\
\hline \multicolumn{3}{|c|}{\begin{tabular}{l|l|l} 
& \multicolumn{1}{|c|}{ Mucio meramu } & \multicolumn{1}{|c|}{ Mervicio. }
\end{tabular}} \\
\hline Pluralismo. & Relaciones competitivas. & Relaciones cooperativas. \\
\hline Nuevas relaciones de prestación. & Clientelización. & Participación. \\
\hline
\end{tabular}

nuevo espacio, en la esfera operativa, para un modelo de administración basado en el servicio, el pluralismo cooperativo y las relaciones de participación (BRUGUé, 1993). Este, además, afirma su propia vinculación normativa con el enfoque de profundización de los roles redistributivos del Estado. El cuadro 3 perfila los dos posibles escenarios alternativos de carácter posburocrático, diseñados a partir de las tres dimensiones fundamentales inscritas en el cambio de paradigma.

\section{La transformación de las relaciones de prestación 5 : emergencia y desarrollo del nuevo modelo consumerista}

\section{La realidad de partida: insensibilidad administra-} tiva y dominación burocrática

Tras desarrollar una panorámica analítica general, este apartado centrará la linea argumental en uno de los aspectos centrales y más ampliamente tratados del cambio de modelo administrativo a lo largo de los ochenta: el carácter de las relaciones que se establecen entre la Administración y las personas en el proceso de prestación de servicios. Dichas relaciones pueden revestir características muy diversas. El tipo de relación dependerá del modelo predominante de regulación y gestión pública, y se definirá, más en concreto, en cada uno de los ámbitos de relación que ese modelo establezca con las personas.
La producción y recepción de servicios públicos en el contexto socioburocrático debe entenderse a partir de su inserción en una doble coordenada: el avance del rol social del Estado, expresado en la extensión y profundización de campos de actividad pública; y el predominio simultáneo de una cultura político-administrativa cerrada tanto a la diferenciación personal de las necesidades, como a la interacción, individual o colectiva, en los procesos de prestación. Ello genera, en primer lugar, un sistema administrativo tendente a la estandarización, fuertemente insensible e introvertido, con poca o nula capacidad de recepción, aprendizaje y adaptación (STOKER, 1989). Y, en segundo lugar, el predominio del componente tecno-profesional, el cual induce a procesos de prestación unidireccionales, donde los ciudadanos juegan roles pasivos de simples receptores de servicios, sometidos a relaciones desiguales de dominación burocrática (DunlEAVY, 1980). En sintesis, la experiencia vital de las personas pasa a depender, progresivamente y en multitud de campos, de la actividad de la Administración ${ }^{6}$. Al mismo tiempo que ésta les trata como ciudadanos menores de edad, que se limitan a recibir pasiva y obedientemente la amplia gama de servicios que les son ofrecidos.

La Administración burocrática genera unas relaciones de prestación marcadas por la pasividad, la dependencia y la sumisión de los administrados. Dichas relaciones se desarrollan en ámbitos diversos, en los cuales pueden adquirir matices y configuraciones específicas. ROSENBLOOM (1986) distingue tres ámbitos: el económico-fiscal, el social y el político. En la Dimensión Económico-Fiscal, el Estado keynesiano asume una capacidad sin precedentes de gestión y dirección pública. Las relaciones que se establecen con las personas en ese contexto se inscriben plenamente en la lógica general. Los administrados se convierten en sujetos pasivos y atenazados del doble juego imposición-protección. Imposición pública cuando 
se reclaman recursos y se prescriben sacrificios; protección paternalista cuando se reciben beneficios. En su Dimensión Social, la Administración del EBK recoge el relevo de la familia, la comunidad, las organizaciones religiosas y el mercado en un amplio abanico de actividades relacionadas con la reproducción social de la población. Las personas se relacionan con el sector público para recibir asistencia, rentas mínimas, bienes básicos 0 incrementar su bienestar. Tal como observa Hummel (1977), el modelo organizativo jerárquico, formalista y profesionalizado que caracteriza a dicha dimensión administrativa se traduce en unas relaciones unidireccionales, impersonales y distantes con sus administrados. La gestión socioburocrática acaba adoptando una lógica tendente no a solucionar necesidades sino simplemente a procesar problemas. Finalmente, en la Dimensión Políti$\mathrm{ca}$, las relaciones entre las personas y la esfera pública vienen cruzadas por nuevas tensiones. MOSHER lo formula en estos términos:

"Cómo puede organizarse un servicio público para operar de forma compatible con los principios democráti$\cos$ ? ¿Cómo se puede asegurar que un cuerpo tan diferenciado como el de los empleados públicos actuará en interés de toda la comunidad, será un instrumento para el conjunto de la colectividad?" (1968, p.4).

Observando lo que ha sucedido en la práctica, resulta fácil concluir que se ha producido un desequilibrio en favor de la racionalidad administrativa: el proceso de burocratización ha debilitado la capacidad de actuación de los políticos electos. El tecno-profesionalismo ha generado despolitización en la esfera representativa y ello se ha traducido en una fuerte pérdida de control de los ciudadanos sobre su sistema político.

2. ¿Qué cambia y bacia dónde?: alcance y características del paradigma consumerista.

A lo largo de los ochenta el modelo pre-existente de relaciones entre la esfera pública y las personas entra en crisis en todas y cada una de sus dimensiones. Como se verá más adelante en profundidad, GYFORD (1991) centra el cambio en la noción teórica de activación de roles, lo cual le permite sostener la necesidad de redefinir los papeles clásicos pasivos de "contribuyente, receptor y votante" (1991: 8-21) en los nuevos de "accionista, consumidor y ciudadano" (1991: 153-179), válidos para cada una de las dimensiones tratadas anteriormente. Sin embargo, durante los años ochenta, la praxis de la reactivación de roles se ha situado de forma casi exclusiva en el terreno de la prestación de servicios -la dimensión social de ROSENBLOOM-, excluyendo sistemáticamente las dimensiones económico-fiscal y política. Es precisamente la exclusión de la dimensión política lo que vendrá a adquirir centralidad en nuestra argumentación posterior, y lo que nos permitirá reubicar el proceso de clientelizaciôni: del campo de las modas organanizativas al de las opciones ideológicas. Ahora, sin embargo, el análisis se centrará en la transformación de las relaciones de prestación. Veremos, en ellas, como el nuevo paradigma consumerista se abre paso. Más tarde, al explicitar sus límites, se retomará la crítica político-ideológica.

Los factores explicativos del cambio de raíz consumerista en las relaciones de prestación son múltiples. Como elemento habilitador, cabe destacar la traslación al sector público de las transformaciones tecnológicas recientes. Con ellas, se posibilita la desestandarización y las interacciones con el público. El elemento determinante, sin embargo, hay que situarlo en el campo sociocultural. La fragmentación de la estructura social se traslada a la esfera del consumo, en ella, se empiezan a afirmar valores de diversidad y pluralismo, así como la activación de roles individuales y colectivos. En la medida en que una parte importante de las relaciones de consumo sigue teniendo lugar en el sector público, la personalización y las actitudes de afirmación de roles pasan a tener uno de sus principales frentes de expresión en la reacción contra los modos estatistas y burocráticos de provisión pública? En consecuencia, se abre paso a cambios que van dando lugar a un enfoque global consumerista, en el campo de las Administraciones Públicas.

El modelo consumerista se ha ido configurando en la práctica a partir de un abanico amplio de iniciativas puntuales, cuyo nivel concreto de éxito ha permitido diferentes grados de generalización y solidez en las Administraciones Públicas. Dos notas definitorias enmarcan y otorgan coherencia conceptual al conjunto de estas iniciativas: En primer lugar, su ámbito se ciñe a la dimensión de prestación de servicios; el proceso de diseño de políticas - la esfera sustantiva queda pues al margen. En segundo lugar, su origen se encuentra, mayoritariamente, en las técnicas desarrolladas en el sector privado; se da, por tanto, un proceso de mimetismo que tiende a difuminar las bipotéticas diferencias entre gestión privada y pública.

En el marco de esas notas definitorias, las principales iniciativas del modelo consumerista pueden ser ordenadas bajo dos grandes categorías tipológicas ${ }^{8}$ :

- El getting closer (iniciativas de dentro hacia fuera). Es decir, la aproximación de la Administración a sus públicos reales y potenciales, en una línea de mayor sensibilidad y receptividad a una estructura individualizada de necesidades. En este marco, toman sentido iniciativas y experiencias tales como la descentralización funcional, 
los nuevos roles de los front-line (técnicos de base), la priorización de las áreas de contacto ${ }^{9}$, el conocimiento de necesidades o la mejora de las estrategias de comunicación, accesibilidad y receptividad.

- El learning from (iniciativas de fuera hacia dentro). Es decir, el impulso a una implicación más activa y directa de los propios usuarios en los procesos de servucción. Aparecen aquí las prácticas de coproducción de servicios, de implicación de los clientes en tareas específicas, los mecanismos deliberativos y de consulta, y los sistemas de petición, queja y reclamación.

Podemos afirmar que ambos aspectos configuran el núcleo del modelo consumerista, construido como aproximación global al conjunto de cambios en las relaciones de prestación ${ }^{10}$.

3. El proceso de consumerización de las relaciones de prestación: una propuesta de interpretación analítica.

A partir de estas categorías, puede desarrollarse una propuesta de interpretación analítica, que posibilite ubicar a las Administraciones Públicas en función del nivel de plasmación real de los principios consumeristas. La Dimensión I del Cuadro 4 -eje horizontal- propone la división de los principios del getting closer en dos variables operativas. Por una parte, el nivel de extroversión o sensibilidad alcanzado por el proceso de servucción pública, medido a través de indicadores de capacidad de aprendizaje y respuesta. Por otra, la naturaleza de la relación entre gestor, público y clientes, en términos de posiciones de poder relativo; desde relaciones de dominación de base tecno-profesional a relaciones simétricas o de igualdad. La Dimensión II, por su parte, indica el nivel de implicación, y por lo tanto de desarrollo de roles activos, por parte de los clientes (priorizando, pues, el aspecto central del learning from); desde interacciones intensas, plasmadas en procesos de coproducciọn o cogestión a implicaciones elementales o roles pasivos. Los niveles de consumerización vendrán expresados por el alcance del desplazamiento en la dirección marcada por ambos vectores del cuadro. En síntesis, la transformación de unas relaciones de prestación pasivas, introvertidas y de dominación en otras coproductivas, sensibles e igualitarias expresarían el cambio hacia el paradigma posburocrático en su concreción consumerista.

\section{Límites y alternativas al modelo consumerista}

Al inicio del apartado anterior, se enmarcaba conceptualmente el consumerismo en la dimensión administrativa y en la recepción de técnicas gestoras del sector privado. Ambos aspectos marcan con toda precisión los límites del modelo consumerista y perfilan los ejes argumentales sobre los cuales se han construido, en la década de los noventa, las principales críticas a éste.

\section{La especificidad de la gestión pública.}

Un primer eje de crítica al consumerismo de los años ochenta se desarrolla al filo de su carácter mimetizador de las iniciativas de la gestión privada. En opinión de HAMBLETON (1988), las importaciones organizativas y gestoras del mundo de los negocios generan dificultades de adaptación a las peculiaridades del sector público, las cuales se manifiestan en dos direcciones.

En primer lugar, los clientes del sector privado disfrutan de un poder de elección que no tiene equivalente en el ámbito público. Las instituciones públicas descansan sobre unos fundamentos políticos que condicionan sus relaciones con los administrados ": sus servicios no han

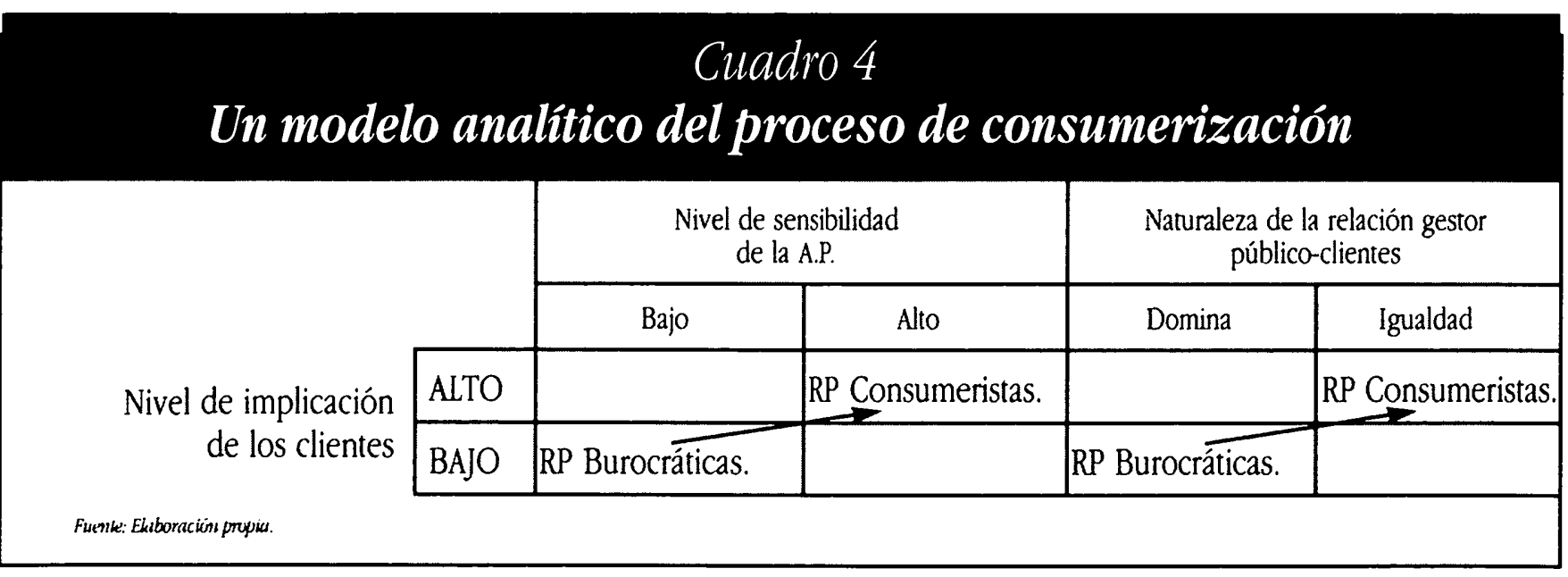


de satisfacer únicamente demandas individuales sino, sobre todo, intereses colectivos. Además, muchos de estos servicios se ofrecen en régimen de monopolio y, por lo tanto, el consumidor puede ejercer la capacidad de control que le brinda el sistema electoral, pero no una elección investida de poder real como la que permite el mercado (Mendoza, 1990). En palabras de Subirats (1989), nos trasladamos de la lógica "una decisión-un actor" a una situación donde las decisiones se toman a través de interacciones entre individuos, grupos e instituciones. Este último punto nos conduce al segundo problema: las opciones estrictamente empresariales tratan a sus clientes como personas aisladas con las que establecen relaciones mercantiles puntuales, mientras que el sector público ha de tratarles como grupos con necesidades colectivas. Los modelos de relación, consecuentemente, son más complejos y requieren de unos instrumentos que no ofrece el marketing privado.

A través del reconocimiento de estas dificultades ha aparecido un grupo de autores que reivindican la necesidad de superar el mimetismo acrítico que ha dominado los años ochenta, reivindicando la "sustitución de la imitación por la innovación" (METCALFe, 1993) o la necesidad de integrar la dimensión colectiva en las relaciones que se establecen entre la Administración y sus administrados (Clarke y Stewart, 1987; Stewart, 1988).

De este modo, en la voluntad de construir una gestión pública (GP) independiente de las experiencias privadas, se reafirma la necesidad de una dirección política que no sirva únicamente para optimizar el funcionamiento de instituciones concretas, sino también para orientarlas en el entramado de organizaciones que conforman el complejo mundo de la GP actual. La Administración Pública moderna, en palabras de OSBORNE y GAEBLER (1994), no puede limitarse a "remar", es decir, a implementar correcta pero asépticamente una batería de políticas públicas, sino que también debe "llevar el timón", conducir la nave hacia la consecución de unos objetivos que sólo pueden ser definidos políticamente. METCALfE (1993) utiliza términos distintos para proponer una distinción similar. Existe, en su opinión, un nivel de micro gestión donde lo prioritario es mejorar la mecánica de funcionamiento de cada organización, y un segundo nivel de macro gestión donde la preocupación no se centra en los medios sino en los objetivos. Durante los ochenta, la micro gestión pública ha aprendido del sector privado, pero esto ya no parece suficiente para los noventa. En un entorno que tiende a la dispersión y al cambio, es necesario desarrollar habilidades estratégicas que nos permitan dirigir y controlar la incertidumbre. Estas habilidades pueden tomar formas diferentes pero con un denominador común: la repolitización de la gestión pública, es decir, la introducción de unos valores y de un diseño estratégico de las actuaciones que faciliten timonear la nave en una dirección predefinida.

Creemos que las relaciones entre la Administración y los administrados deben replantearse a partir de la comprensión de este proceso de repolitización. Si únicamente nos ocupamos de la micro gestión -de tensar los músculos para "remar" mejor-, es muy probable que una relación mercantil como la que establece una empresa con sus clientes sea el camino más adecuado para su mejora. Sin embargo, la GP está obligada a operar a un nivel macro, a "llevar el timón" hacía el logro de unos objetivos políticos que, en una sociedad democrática, han de definirse a partir de la participación de los ciudadanos.

El término "participación" se relaciona con la reactivación del rol de los administrados a la que antes nos referíamos, pero desborda el ámbito estrictamente prestacional que normalmente se asocia al proceso de mercantilización de la relación Administración-administrados. Ya no se trata únicamente de satisfacer las demandas de unos consumidores, sino de relacionarse con unos ciudadanos que deben adherirse a un determinado proyecto ideológico, expresar su visión del interés colectivo y legitimar activamente los objetivos propuestos por los responsables políticos. Estas afirmaciones nos conducen a la segunda crítica al modelo consumerista.

\section{La unidimensionalidad del modelo consumerista.}

En otro plano argumental, GYFORD (1991) pone sobre la mesa un segundo interrogante en relación al modelo consumerista: el carácter aparentemente avalorativo derivado de su inscripción en la esfera operativa (prestacional) del Estado.

En el enfoque crítico de GyFord se reconoce la dimensión administrativa como uno de los terrenos de relación de las personas con el Estado. En éste, se pueden llevar a cabo estrategias de cambio tendentes a promover roles personales activos en los procesos de prestación; adquiere aquí sentido el concepto de cliente $y$, por extensión, el modelo consumerista. Sin embargo, GYFORD propone otras dos dimensiones en las cuales los roles tradicionales son también susceptibles de transformación. En concreto, la expresión de preferencias personales en la esfera política ha quedado ceñida tradicionalmente al comportamiento electoral. La activación de roles políticos pasaría por la expresión sistemática de dichas preferencias en los procesos de diseño de políticas públicas; el concepto de ciudadano adquiere así su máxima significación. Por último, existe una dimensión 


\begin{tabular}{|c|c|c|c|}
\hline \multicolumn{4}{|c|}{$\begin{array}{c}\text { Cuadro } 5 \\
\text { Modelos de relación entre el sector público } \\
\text { y las personas }\end{array}$} \\
\hline \multirow[b]{3}{*}{ Nuevos roles personales activos. } & Esfera política & Esfera administrativa & Esfera fiscal \\
\hline & Modelo ciudadano & Modelo consumerista & Modelo accionarial \\
\hline & Ciudadano. & Cliente. & Accionista. \\
\hline Concepción de las personas. & Colectiva. & Individual. & Individual. \\
\hline $\begin{array}{l}\text { Carácter de la implicación perso- } \\
\text { nal. }\end{array}$ & Participativo. & Consultivo reclamatorio. & Reclamatorio. \\
\hline Valores político-ideológicos. & Nueva izquierda. & Reformistas. & Nueva derecha. \\
\hline
\end{tabular}

fiscal en la cual el cambio actitudinal consistiría en el paso de contribuyentes pasivos -simples fuentes de recursos fiscales- a accionistas o acreedores del Estado, con derecho a reclamar de éste la protección efectiva de sus intereses (ver cuadro 5).

La triple distinción de GYFORD es relevante en la medida que:

i) La opción por uno de los tres modelos-accionarial, consumerista o ciudadano- determina el carácter $y$ los limites de las nuevas relaciones entre las personas y el Estado. Unicamente la opción por el modelo ciudadano como estrategia de cambio conduce a procesos de naturaleza plenamente participativa. En cambio, el modelo consumerista limita los procesos de implicación personal en la relaciones de prestación a un estadio predominantemente consultivo-reclamatorio.

ii) La opción por uno u otro modelo reviste carácter normativo; es, en definitiva, el correlato de posiciones de carácter político-ideológico. Así, el modelo ciudadano viene a corresponder a los valores de la nueva izquierda, el consumerista estaría conectado a orientaciones reformistas, y el accionarial, por último, sería consistente con la cultura de la nueva derecha.

En síntesis, GyFord vuelve a situar el modelo consumerista ante sus contenidos y sus límites político-ideológicos. Se argumenta que la ubicación explícita de éste en la esfera operativa no prescribe un carácter normativamente neutro del modelo. Su naturaleza valorativa, en cambio, se desvela en dos aspectos: a) la plena desconexión del consumerismo respecto de valores políticos de profundización de la ciudadanía, y b) el desarrollo de unas relaciones de prestación circunscritas a la dimensión individual de cliente con implicaciones activas de alcance limitado. En la siguiente sección repasaremos el desarrollo de este debate en el contexto británico, donde se originó.

3. El debate británico.

Tal como hemos indicado en el segundo apartado, en el proceso de construcción del EBK, la Administración Pública se ha basado en la articulación de enormes y profesionalizadas estructuras burocráticas. Este tipo de organización ha facilitado la universalización de los servicios públicos, pero también ha propiciado la aparición de una serie de problemas que hemos condensado en las ideas de insensibilidad administrativa y dominación burocrática: en breve, el conocido paternalismo burocrático.

El paternalismo burocrático puede interpretarse como la perversión del modelo de prestación de servicios públicos típico del EBK y, simultáneamente, como un punto de partida común para la serie de alternativas que se fueron edificando a lo largo de la década de los ochenta. Un diagnóstico común, sin embargo, no implica un tratamiento igual del problema.

Analicemos, pues, el origen y evolución de estas alternativas en el contexto político de Gran Bretaña.

La primera opción está íntimamente asociada a las propuestas de la Nueva Derecha (ND) y empezó a concretarse a partir de 1979, cuando Margaret Thatcher fue elegida Primera Ministra. La ND se caracteriza por rechazar la notoriedad de los grupos sociales organizados que representan intereses colectivos y por adoptar una visión 
racional-egoista de las personas. Consecuentemente, ha impulsado procesos mercantilizadores y ha introducido la competencia entre las diversas Administraciones Públicas involucradas en lá presiación dét servicious púbulicos (ForsYTH, 1980; Butcher et al., 1990). Se trata de una posición ideológica que niega la existencia del interés colectivo (siempre que no sea interpretado como la suma de intereses individuales) y que sitúa las relaciones Administración-administrados en un contexto estrictamente económico-prestacional. En otras palabras, el tipo de relación entre la Administración y sus administrados debería ser la misma que se establece en el sector privado entre clientes y proveedores.

A las formulaciones de la ND -de claro contenido políico conservador- se les opuso una segunda alternativa ideológica, la cual pretendía tanto defender la provisión pública de los servicios como promover fórmulas de prestación más democráticas. Según los partidarios de esta alternativa, agrupados bajo el rótulo de Nueva Izquierda Urbana (NIU) ${ }^{12}$, la solución al paternalismo burocrático sería una extensión de la democracia hasta nuevos ámbitos de decisión (por ejemplo, los barrios). La NIU pone el acento en la capacidad de decisión y de actuación de los propios administrados en tanto que ciudadanos y no únicamente como clientes. En este sentido, sus propuestas tienden a delegar y descentralizar el poder que monopolizó la Administración en el EBK. El interés público no sólo existe, sino que además puede ser promocionado por los propios interesados sin la intervención directa y constante de los representantes políticos o de las autoridades administrativas (GYFORD, 1985; BODDY y FuDGE, 1984). No se trata sólo de escuchar a los ciudadanos sino de involucrarlos en la solución de sus propios problemas (empowerment).

La tercera gran opción tuvo su origen en las dificultades reales que sufría la prestación de servicios públicos bajo la presión del modelo thatcherista ya establecido. A partir de mediados de los años ochenta, emerge progresivamente la alternativa conocida bajo la etiqueta de consumerism. Debemos ser cautelosos en la conceptualización de este enfoque, puesto que bajo su manto se encasillan iniciativas muy diferentes: la plasmación británica del consumerismo propiamente dicho y la denominada corriente del Public Service Orientation (PSO) (LGMB 1988, 1989).

En Gran Bretaña, el consumerismo pasa también por la introducción en el campo de la gestión pública de iniciativas, métodos y mecanismos propios del mundo privado; pero, a diferencia de las propuestas de la ND, manteniendo la preeminencia de la prestación pública de bienes y servicios. La caracterización del sujeto perceptor de servicios como consumidor intenta resaltar el desequilibrio que existe entre los proveedores y los perceptores de bieñes y servicios cin favoú de los primeros. Para cambiar esta relación de poder es necesario, según PotTer (1988), fomentar cinco factores: acceso, elección, información, reequilibrio y representación. Es decir, el Consumerismo consistiría en fomentar el acceso de los consumidores a una gama de productos y servicios lo más amplia posible para que pudieran elegir razonablemete entre las diversas opciones. A tal efecto, una información veraz, así como la articulación de canales de comunicación de sus quejas hacia los productores de servicios, serían imprescindibles. Finalmente, una adecuada representación de los intereses de los consumidores frente a aquéllos que toman decisiones que afectan a su bienestar, significaría reequilibrar la relación entre proveedores y consumidores a favor de los últimos.

En segundo lugar, las iniciativas del PSO conllevan un esfuerzo, desde el propio sector público, por desarrollar una serie de mecanismos propios para mejorar la gestión. Es decir, al igual que en el caso del Consumerismo en sentido estricto, se mantiene la preeminencia de la prestación pública, pero se reconoce que el sector privado no puede ser la única fuente de inspiración modernizadora, debido al carácter cualitativamente distinto entre ambos (RANSON y STEWART, 1988). A diferencia de las propuestas de la NIU, el papel de los grupos y de los ciudadanos no ha de comportar necesariamente la reducción del rol de los representantes políticos y de las autoridades administrativas, puesto que pueden desarrollar un papel estratégico de primer orden. El PSO, por lo tanto, es un intento de inculcar en las Administraciones Públicas un cambio significativo en su cultura organizativa. Este cambio puede sintetizarse en una expresión: las Administraciones Públicas prestan servicios "para el público" y no "al público" (providing services for the public and not providing services to the public). De esta manera, la organización administrativa dejaría de ser autocentrada y ensimismada -características típicas del paternalismo burocrático- para pasar a ser abierta y sensible a las necesidades del público.

La opción del PSO ha sido calificada como tecnocrática, ya que, a diferencia de lo que sucedía con las alternativas anteriores, su ubicación político-ideológica es esquiva. A grandes rasgos, sin embargo, las opciones consumeristas corresponderían a los partidarios del Gobierno Comunitario (GC) ${ }^{13}$, aunque la conexión no sea tan clara como en los casos de la ND y la NIU. Los partidarios del GC reconocen el papel de los grupos sociales en la formación de la agenda de actuación 


\begin{tabular}{|c|c|c|}
\hline \multicolumn{3}{|c|}{$\begin{array}{c}\text { Cuadro } 6 \\
\text { Tres conceptos de ciudadania } \\
\text { según Walsh y Prior }\end{array}$} \\
\hline Concepto de ciudadanía & Valores dominantes & Modelo de Estado \\
\hline Poseedor de derechos individuales. & Libertad, elección, individualismo. & Estado mínimo, protector y fuerte. \\
\hline Súbdito de un Estado. & Universalismo, obligación, autoridad. & $\begin{array}{l}\text { Estado centralizado, representativo y } \\
\text { fuerte. }\end{array}$ \\
\hline Miembro de una comunidad. & Comunitarios, solidarios, colectivistas. & $\begin{array}{l}\text { Estado descentralizado, participativo } \\
\text { y débil. }\end{array}$ \\
\hline
\end{tabular}

pública y en la gestión de servicios, a la vez que se muestran reticentes respecto a su capacidad de decisión. Prefieren que las autoridades públicas se reponsabilicen de dirigir, impulsar y coordinar las diferentes iniciativas, aunque reivindicando siempre tanto la vocación de servicio a los ciudadanos como la existencia de un interés colectivo por promover (RAINE, 1981; JONES Y STEWART, 1983; STEWART, 1989).

\section{El Estado, el mercado, la sociedad civil y las personas}

Los recientes debates sobre la modernización de la Administración Pública se han ido transformando progre- sivamente en debates sobre modelos de Estado. Detrás de la utilización de conceptos como cliente, mercantilización, participación o ciudadanía, encontramos valores que afectan la manera de entender la actividad política, la organización gubernamental y la imbricación de ambas con la sociedad civil y el mercado: el A-B-C de cualquier discusión político-ideológica.

Nos proponemos abordar este debate desde la perspectiva de las relaciones entre el sector público y las personas, considerando que el contenido y la forma de estas relaciones condiciona nuestra percepción de la política, de la Administración y de sus posibilidades de transformación. Las cada vez más frecuentes estrategias propuestas para mejorar el funcionamiento del sector público se han planteado siempre en función de una determinada concepción de los individuos. Más o menos dirección, más o menos participación o más o menos mercantilización son decisiones que se toman después de conectar

\begin{tabular}{|c|c|c|c|c|}
\hline \multirow[b]{2}{*}{ Estado supermercado. } & Rol de la ciudadanía & Rol de las AA. PP. & Valores dominantes & Forma gobierno \\
\hline & Cliente consumidor. & $\begin{array}{l}\text { Actuar como una em- } \\
\text { presa. }\end{array}$ & $\begin{array}{l}\text { Competencia y eficien- } \\
\text { cia. }\end{array}$ & Supermercado. \\
\hline Estado de servicios. & Consumidor ciudadano. & Actuar receptivamente. & Receptividad y servicio. & Estratégico. \\
\hline $\begin{array}{l}\text { Estado de autogobierno } \\
\text { social. }\end{array}$ & $\begin{array}{l}\text { Ciudadano coproduc- } \\
\text { tivo. }\end{array}$ & $\begin{array}{l}\text { No actuar, delegar, ca- } \\
\text { pacitar. }\end{array}$ & $\begin{array}{l}\text { Autodesarrollo y parti- } \\
\text { cipación. }\end{array}$ & Descentralizado. \\
\hline
\end{tabular}


las formas de organizar el gobierno de una sociedad con la naturaleza de sus miembros.

En este sentido, WalSH y Pror (1992) nos proponen una distinción entre tres concepros de ciudadanía y ures modelos de Estado (cuadro 6).

Esta tipología resulta útil para vincular los conceptos de ciudadanía y Estado con diferentes formas de participación. Según WaLSH y PrIOR, el súbdito de un Estado asume la distancia de la representatividad, se somete al ejercicio del poder político y desarrolla actitudes eminentemente pasivas y victimistas. El poseedor de derechos individuales, en cambio, actúa como un comprador en el mercado; mira, escoge, paga y se somete a los dictados de la publicidad. Finalmente, el miembro de la comunidad reivindica su participación activa en la definición de objetivos políticos, así como su capacidad de perseguir intereses no sólo individuales sino también colectivos.

Analizando la situación británica, WALSH y PrIOR llegan a la conclusión de que el escenario dominante durante la segunda mitad del siglo $\mathrm{xx}$-donde el ciudadano era un súbdito obediente en el marco de unos Estados de Bienestar fuertes, centralizados y representativos- está siendo sustituido por un proceso de mercantilización de la política. Es decir, el ciudadano se convierte progresivamente en un poseedor de derechos individuales que intenta satisfacer sus demandas en el mercado. El Estado, paralelamente, reduce su capacidad de intervención, a la vez que defiende con fuerza los intereses individuales de cada uno de sus miembros. Sin embargo, tal como apuntan los propios autores, existe otra alternativa que combina la presencia de una ciudadanía consciente de su pertenencia a la comunidad con un Estado más débil, participativo y descentralizado. En esta tercera opción, el protagonismo en la regulación de los intereses colectivos pasa del Estado (perspectiva Estado-céntrica tradicional) a la sociedad civil (perspectiva socio-céntrica), mientras que en el caso anterior se trasladaba al mercado (perspectiva mercado-céntrica).

En opinión de otros autores, como por ejemplo JoRGENSEN (1993), el debate actual sobre el modelo de Estado que se impondrá tras la crisis del EBK recoge precisamente las tres perspectivas a las que antes nos referíamos: la Estado-céntrica, la socio-céntrica y la mercado-céntrica. Se parte, por lo tanto, de una premisa según la cual la autoridad pública, el mercado o la sociedad civil son las tres únicas formas posible de regulación social. A partir de esta convicción, JORGENSEN distingue entre el Estado de servicios (perspectiva Estadocéntrica), el Estado supermercado (perspectiva mercado-céntrica) y el Estado autogobernado (perspectiva socio-céntrica) (cuadro 7).

Podemos reinterpretar algunas de las aportaciones que hemos expuesio, intentiandu conintiñar el coñcépió de ciudadanía con el debate político-ideológico que viene caracterizando la década de los noventa. Según parece, nos encontramos ante un proceso de reactivación del rol de los ciudadanos que pone en enormes dificultades la continuidad del modelo tradicional de Estado representativo, burocratizado y centralizado. Al mismo tiempo, emergen alternativas que, en cualquier caso, han de ser capaces de dar respuesta (responsive state) a unas personas con vocación y capacidad para reivindicar la satisfacción de sus necesidades, en contextos tanto políticos como fiscales o prestacionales.

La concreción de esta capacidad de respuesta se puede articular en escenarios diferentes en función de la primacía del mercado, de la autoridad política o de la sociedad civil. Sin embargo, y ahora estamos exponiendo nuestra opinión, consideramos que estas tres alternativas son reducibles a dos. Por una parte, la perspectiva de autorregulación de la sociedad civil -más allá de los mecanismos de mercado- requiere, en el marco de sociedades complejas, de mecanismos estratégicos de autoridad pública. De este modo, en el espacio de encuentro -aún conflictivo y dialéctico- entre el Estado y la sociedad civil podemos situar el polo de la regulación pública. En el otro extremo, ubicamos la alternativa de una sociedad mercado-céntrica, en la cual se da el predominio de los mecanismos de regulación mercantil, por encima de la participación social y sin espacio para roles públicos directivos. Así pues, creemos que la repolitización de nuestras sociedades debe asumirse sin tapujos.

El auge de la sociedad civil podría, en cambio, estar maquillando un proceso de mercantilización de las relaciones sociales que no se atreve a aflorar abiertamente. También podría suceder que la alternativa socio-céntrica estuviera configurando el espacio de consenso que ha venido reclamando la confianza democrático-capitalista en el fin de las ideologias. Esta confianza ha perdurado hasta finales de los ochenta, pero hoy en día ya se acepta la necesidad de repolitizar el análisis de nuestras sociedades. Y nosotros creemos que esto debe hacerse, reconociendo la necesidad de recuperar -aunque redefiniendo- conceptos clásicos como los de izquierda y derecha; por mucho que lo lamenten nuestros políticos, peleando durante décadas por ocupar un centro dominado por la indefinición y una apariencia casi médica de objetividad. En el caso que nos ocupa, no se trata de defender o de atacar los mecanismos de mercado, sino 


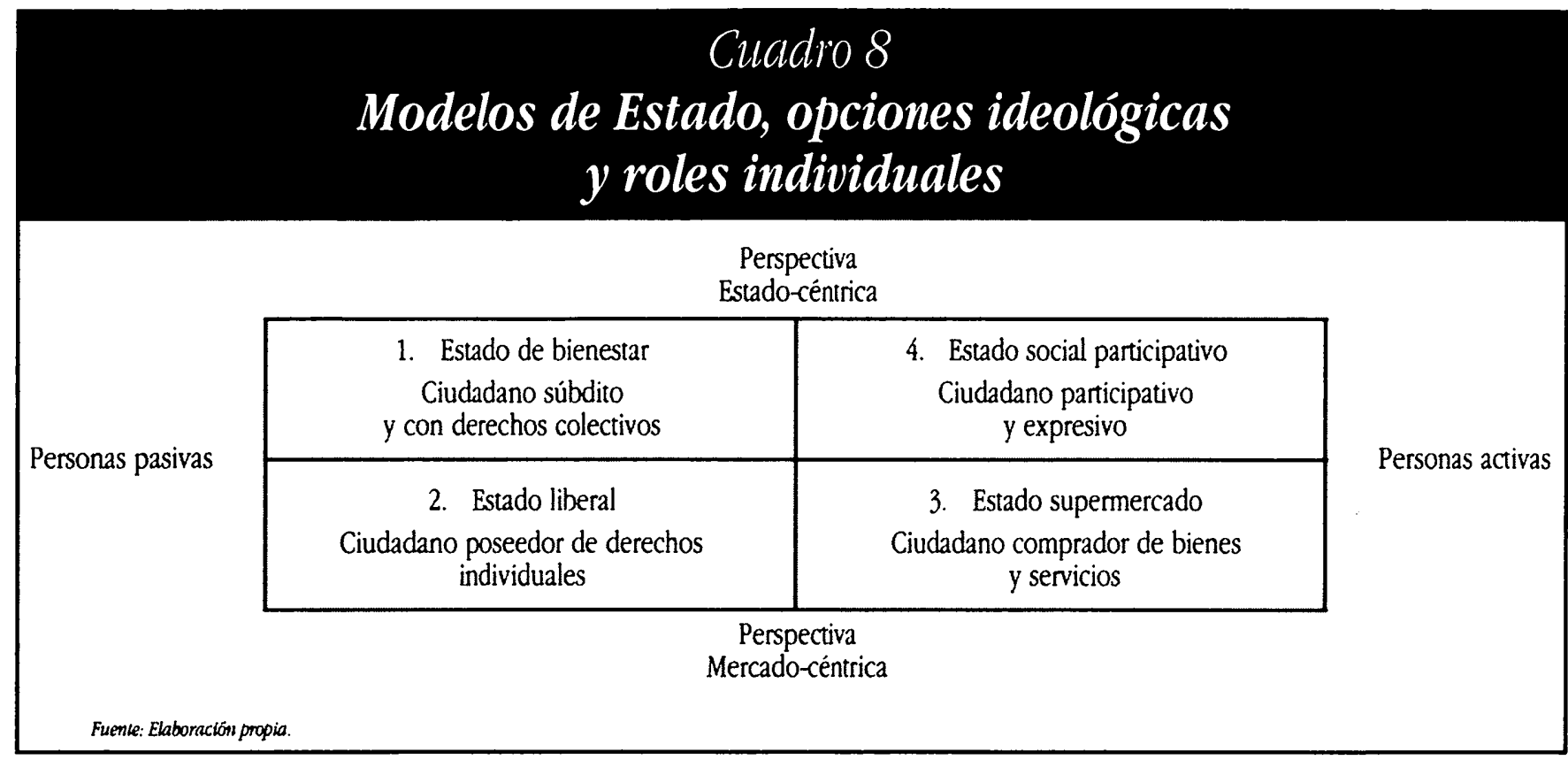

de reconocer que tras cada defensa y cada ataque hay argumentos políticos. El proceso de mercantilización de las relaciones entre la Administración y sus administrados no es una moda sino una opción ideológica, y como tal hay que tratarla.

Proponemos, a modo de conclusión, un modelo analítico que sirva para reinterpretar el debate que hemos presentado en este artículo. Tomamos como punto de partida la reactivación del rol de los ciudadanos, mientras que empleamos la dicotomía público-privado (perspectivas Estado y mercado-céntricas, respectivamente) para referirnos a las opciones ideológicas que nos permiten situar esta reactivación lejos del plano estrictamente técnico-organizativo. Situando las dos variables en un eje de coordenadas, obtenemos cuatro escenarios ideales representados en el cuadro 8.

El Estado de Bienestar keynesiano y el Estado Liberal resumen las dos opciones políticas más utilizadas por las democracias capitalistas durante el siglo xx. En ambas, los administrados muestran una actitud pasiva frente a la Administración y al gobierno, mientras que sus discrepancias se sitúan en el terreno político-ideológico. Tal como han apuntado diversos autores, la democracia representativa y la organización burocrática son los artífices de la pasividad ciudadana, ya que se trata de mecanismos que fomentan un estilo de relación jerárquica, formalista y distante: las personas se convierten en casos sin nombre que únicamente encuentran solución a sus problemas a través de procesos de generalización. La ciudadanía no tiene preocupaciones personales, sino que las adecúa a la percepción que de ellas tienen los representantes políticos y los responsables administrativos. El administrado, tal como observaba RosenBlOOM (1986), es sujeto, no objeto, de la acción pública.

Desde este punto de partida, el Estado de Bienestar keynesiano y el Estado Liberal se diferencian por el contenido y el alcance de sus intervenciones, es decir, por su mayor o menor vocación de regulación pública. Esta ha sido, precisamente, la distinción más clara entre las opciones políticas tradicionales: la derecha tiende a potenciar la regulación privada y a reducir el tamaño de la Administración, mientras que la izquierda prefiere impulsar la regulación pública e incrementar la capacidad de intervención sobre la sociedad. El administrado es pasivo en ambos casos, pero en el primero es capaz de generar interés colectivo a través de la maximización de sus preferencias individuales, mientras que en el segundo cede a una organización pública la responsabilidad de definir y defender un interés público independiente de la suma de los intereses personales. Observamos, por lo tanto, como distintas percepciones sobre la naturaleza de las personas generan posturas político-ideológicas dispares, aun bajo un modelo institucional regido por reglas comunes como la democracia representativa y la Administración burocrática.

Los años ochenta y, en especial lo que llevamos de los noventa, han provocado, si no la crisis, sí un serio replanteamiento de los modelos representativo y burocrático tradicionales. Una de las manifestaciones más destacadas de este proceso la encontramos en la reactiva- 
ción del rol de las personas, tanto en el contexto político como en el fiscal y el prestacional. Esta reactivación conlleva lo que GrFord (1991) ha calificado como la "reestructuración de los instrumentos públicos de producción y de relación", lo que OSBORnE y GaEBLER (1994) denominan la "reinvención del gobierno", lo que Koolman (1993) analiza bajo la etiqueta de "nuevos modos de gobernación o lo que, en definitiva, podríamos resumir como un proceso de redefinición de las formas clásicas de gobernar y gestionar nuestras sociedades.

Durante cierto tiempo se pensó que este proceso de cambio era unidireccional, que habíamos superado las viejas polémicas ideológicas y que conceptos tradicionales como los de izquierda y derecha habían perdido todo su contenido. En este artículo, sin embargo, hemos intentado argumentar que una observación más detallada de estas transformaciones pone de manifiesto la existencia de alternativas, las cuales pueden además ser interpretadas en clave político-ideológica. De este modo, la aparición de nuevos modelos de relación entre la Administración y las personas puede analizarse desde diferentes puntos de vista.

En primer lugar, y éste ha sido el observatorio más frecuentado, puede defenderse la aparición de un estilo de relación mercantil: las personas compran bienes y servicios, mientras que la Administración se preocupa únicamente de satisfacer sus demandas de la forma más económica, más eficiente y más eficaz posible. Desde esta óptica, situada en nuestro tercer escenario, se promociona el individualismo y la privatización, ya que se considera que la persecución de intereses privados es la mejor alternativa para generar progreso social. Las semejanzzas con las afirmaciones clásicas de la derecha liberal son más que notorias.

En segundo lugar, existe un escenario alternativo -que hemos llamado Estado Social Participativo- donde se subraya la necesidad de una autoridad pública capaz de promover su propia percepción del interés colectivo, aunque siempre combinándola con el proceso de reactivación de los roles individuales. La autoridad a la que nos referimos, por lo tanto, no puede ser ejercida de forma paternalista ni contando con la obediencia sumisa de sus súbditos, sino articulando mecanismos pluralistas y participativos que permitan un proceso de toma de decisiones más co-producido, más receptivo, más consensuado o, en palabras de MeTCALFE (1993), que sepa combinar una clara vocación participativa con una aproximación top-down a la definición del interés colectivo. Se modifica así el modo de administrar y de gobernar del EBK, pero no se abandona la perspectiva Estado-céntrica que lo caracterizaba.

Hemos identificado, en definitiva, una progresiva transformación del Estado que afecta a sus formas de gobernar, de administrar y de relacionarse con las personas. Sin embargo, en contra de lo que normalmente se cree, también hemos argumentado la persistencia de alternativas político-ideológicas: alternativas que antes se llamaban izquierda y derecha.

\begin{abstract}
- Universitat Autònoma de Barcelona.
' Sobre las variantes tipológicas del Modelo Clásico de Bienestar, THERBORN (1989) distingue entre Estados universales, compensatorios, ocupacionales y residuales, en función del nivel de intervención pública en las esferas social y económica. ESPING-ANDERSEN (1993), en cambio, distingue modelos integrales, corporativos y residuales en función de tipos de incidencia estratificadora y del nivel de desmercantilización.

${ }^{2}$ La crítica a la economía keynesiana se realiza desde tres diferentes corrientes de pensamiento, las cuales companen únicamente su creencia en la optimalidad del libre mercado como mecanismo asignador de recursos: la Escuela Monetarista, la Escuela de las Expectativas Racionales y la Economía de la Oferta. Ver, para una buena síntesis, NEu (1984).

3 Para cada uno de estos tres puntos, pueden consultarse, como textos de referencia, Peters-Waterman (1982), FLYNN-WALS (1988) y OCDE (1991).

- Sobre estrategias alternativas de reestructuración sustantiva del EBK, ver, Rodriguez Cabrero (1992), Garcia Roca (1993) o Gomá (1992).

"Por "Relaciones de Prestación" entenderemos, en adelante, el tipo de relaciones que se establecen entre la Administración Pública y las personas en los procesos de servucción o producción de servicios. FlrnN (1993 cap. 7).

- Para ejemplarizar dicha realidad se suele decir que los ciudadanos de los Estados de bienestar nacen, se educan, se alojan, trabajan, se divierten $y$, finalmente, mueren, bajo algún tipo de tutela administrativa.
\end{abstract}

' Para un desarrollo en profundidad de este argumento, pueden consultarse: Galtung (1984), Johnson (1987), Rodriguez Cabrero (1991), Main (1991) y RODRIGUEZ VILLASANTE (1991).

${ }^{8}$ Ambas categorías son tomadas de los documentos "Getting Closer to the Public" y "Learning from the Public", publicados por el Local Government Training Board, de Gran Bretaña.

"El concepto de "áreas de contacto" se toma de la distinción conceprual de FLYNN (1991 p.149-150) entre core and peripheral services.

${ }^{10}$ Ver, por ej., Hambleton (1988) o Hambleton-Hogcett (1987)

"En adelante, la palabra "administrados" se utiliza sin ningún tipo de connotación conceptual; como simple equivalente de "personas o individuos".

${ }^{12}$ La Nueva Izquierda Urbana es una corriente estrechamente ligada a la izquierda obrera del Partido Laborista y al nuevo radicalismo de clases medias que encuentra acomodo en los gobiernos locales laboristas de importantes ciudades británicas (Liverpool en el primer caso; Londres y Sheffield en el segundo).

is La corriente del Gobierno Comunitario o "Community Government" nace en círculos académicos y de reflexión sobre la práctica político-administrativa municipal en la Inglaterra de los ochenta. Aunque con débiles conexiones partidarias, sus proponentes se situarian cerca de las corrientes moderadas del laborismo. 


\section{Bibliografía}

BARZELAY, M. (1992): Breaking Through Bureaucracy: A new Vision for Managing in Government, University of California Press, Berkeley. BODDY, M. y FuDGE, C. (eds.) (1984): Local Socialism?, Macmillan, Londres. BrucuÉ, Q. (1993): Models de Prestació de Serveis Municipals: Una Anàlisi des de la Ciència Politica, tesi doctoral, U.A.B.

BUTCHER, J. et al (1990): Local Government and Thatcherism, Rouledge, Londres.

Clarke, M. y Stewart, J. (1987): "The Public Service Orientation: Issues and Dilemmas", Public Administration, vol. 65.

DUNLEAVY, P. (1980): Urban Political Analysis, Macmillan, Londres.

Etcock, H.J. (1991): Change and Decay, Longman, Londres.

ESPING-ANDERSEN, G. (1993): Los Tres Mundos del Estado de Bienestar, Ed. Alfons el Magnànim, València.

Fuyns, N. (1991): Public Sector Management, Harvester \& Whestsheaft, Londres.

FLYNN, N. y WALS, K. (1988): Competitive Tendering, INLOGOV, Birmingham.

FLYNN, R. (1993): Marketization in the Public Sector: Implications for Citizenship, professionalization and welfare, ECPR, Limerick.

FORSYTH, M. (1980): Re-Servicing Britain, Adam Smith Institute, Londres.

Galtung, J. (1984): Hay Alternativas, Ed. Tecnos, Madrid.

Garcia Roca, J. (1993): Público y Privado en la Acción Social, Ed. Popular, Madrid.

Gomi, R. (1992): Govern Local y Reestructuració Urbana. Un Model d'Anàlisi de Politiques Públiques, tesi doctoral, U.A.B.

GYFoRD, J. (1985): The Politics of Local Socialism, Allen \& Unwin, Londres.

GrFord, J. (1991): Citizens, Consumers $\&$ Councils, Macmillan, Londres.

Hambleton, R. (1988): "Consumerism, Decentralization and Local Democracy", Public Administration, n. ${ }^{8} 66$

Hambleton, R. y HoggetT, P. (eds.) (1987): Decentralization and Democracy: Localising Public Services, School for Advanced Urban Studies, Bristol.

Hummel, R.P. (1977): The Bureaucratic Experience, St. Martin's Press, Nueva York.

JoHnson (1987): El Estado de Bienestar en Transición, Ministerio del Trabajo, Madrid.

JORGENSEN, T.B. (1993): "Modes of Governance and Administrative Change" en Koiman, J. (ed.), Modern Governance, Sage, Londres.

KING, D.S. (1987): The New Right: Politics, Markets and Citizenship, Macmillan, Londres.

KoOiman, J. (ed.) (1993): Modern Governance, Sage, Londres.

LGTB (1988): Getting Closer to the Public, Londres.

LGTB (1989): Leaming From the Public, Londres.

ManN, K. (1991): "Privatización del Bienestar, Individualismo y Estado", en Rodríguez Cabrero (ed.), Estado, Privatización y Bienestar, Icaria, Barcelona.
Mendoza, X. (1990): "Técnicas Gerenciales y Modemización de la Administración Pública en España", Documentación Administrativa, n.ำ 223.

MetCalfE, L. (1993): "Public Management: from imitation to innovation", en Kooiman, J. (ed.), Modern Governance, Sage, Londres.

Mosher, F. (1968): Democracy and the Public Sector, Oxford University Press, Oxford

MuÑoz DE BusthLo, R. (1989): Crisis y Futum del Estado del Bienestar, Alianza Universidad, Madrid.

NeLL, E. J. (1984): Freee Market Conservatism: A Critique of Theory and Practice, George Allen \& Ullwin, Londres.

OCDE (1991): La Administración al Servicio del Público, MAP, Madrid.

Osborne, D. y Gaebler, T. (1994): La Reinvención del Gobierno, Paidós, Barcelona.

PETERS, T. (1988): Thriving on Chaos, Alfred Knopf, Nueva York.

Peters, T. y Waterman, R. (1982): In Search of the Excellence, Harper \& Row, Nueva York.

PotTer, J. (1988): "Consumerism and the Public Sector: How Well Does the Coat Fit", Public Administration, vol. 66

RaINE, J. (ed.) (1981): In Defense of Local Government, INLOGOV, Birmingham.

Raison, S. y Stewart, J. (1988): "Management in the Public Domain", Public Money and Management, vol. VIII.

Rodriguez Cabrero, G. (ed.) (1991): Estado, Privatización y Bienestar, Ed. Critica, Madrid.

Rodricuez Cabrero, G. (1992): "Fundamentos Teóricos de la Política Social", en Moreno, L. y Pérez Yruela, M. (ed.), Política Social y Estado del Bienestar, Ministerio de Asuntos Sociales, Madrid

Rodríguez VILASANTE (1991): Tendencias y Potencialidades ante una Crisis de Civilización, Mimeo, Madrid.

RosenbloOm, D.H. (1986): Public Administration, Nueva York.

STEWART, J. (1988): Understanding the Management of Local Government, Longman, Londres.

StEWART, J. (1989): "A Future for Local Authorities as Community Government", en Stewan y Stoker (eds.), The Future of Local Government, Macmillan, Londres.

StOKeR, G. (1989): "Creating a Local Government for a Post-Fordist Society: The Thatcherite Project", en Stewart y Stoker (eds.), The Future of Local Government, Macmillan, Londres.

SuBIRATS, J. (1989): Análisis de Politicas Públicas y Eficacia de la Administracion, INAP, Madrid

THERBORN, G. (1989): "Los Retos del Estado de Bienestar", en Muñoz de Bustillo (ed.), Crisis y Futuro del Estado de Bienestar, Alianza Universidad, Madrid.

WaLSH, K. y PrJOR, D. (1992): Citizens and Charters: Developing Service Quality in Local Govermment, ECPR, Limerick. 